\title{
Incidência de near miss neonatal em uma maternidade de médio porte do Nordeste Brasileiro
}

\author{
Incidence of neonatal near miss in a midsize maternity in the Northeast Brazil \\ Anajás da Silva Cardoso Cantalice ${ }^{1}$ (D), Karlla Kellyane Alves Carvalho ${ }^{2}$ (D), Lucian Batista de Oliveira ${ }^{3}$ (D)
}

\begin{abstract}
RESUMO
Objetivo: Verificar a incidência de near miss neonatal (NMN), indicador de "quase morte" por complicações pré ou pós-natais, em uma maternidade de médio porte no Nordeste brasileiro. Modelo do estudo: Coorte retrospectiva, com abordagem indutiva e procedimentos descritivos. Metodologia: Analisou-se prontuários de nascidos no mês de janeiro de 2016, identificando-se os casos de NMN conforme os seguintes critérios: idade gestacional < 32 semanas, recém-nascido (RN) que necessitou de manobra de reanimação ou deprimido, peso ao nascimento $<1500 \mathrm{~g}$, necessidade de cuidados em unidade de terapia intensiva neonatal (UTIN), necessidade de ventilação mecânica, escore de Apgar no $1^{\circ}$ e $5^{\circ}$ minutos < 7. Para verificar a associação entre as variáveis maternas e o diagnóstico de NMN, foi utilizado o teste t de Student. Resultados: Foram avaliados 120 RN. Dentre eles, 26 nascidos de parto normal e 94 de parto cesáreo. A média de idade materna foi de $26,61( \pm 7,9)$. A maioria das genitoras apresentava doenças cardiovasculares $(71,7 \%)$ e $70,8 \%$ pré-natal com número de consultas inferior a sete. Identificou-se uma incidência de $30 \%$ de NMN, sendo a maioria do sexo masculino (55\%). A taxa de internação na UTIN foi de 17,5\%. O menor número de filhos vivos e médias superiores de partos cesáreos apresentaram associação significativa $(p<0,05)$ com o NMN. Conclusão: O indicador de NMN apresentou uma elevada incidência, mesmo em hospital de referência para gestação de alto risco, sendo um método de grande relevância para avaliação e prevenção de morbidades graves do RN.
\end{abstract}

Palavras-chave: Neonatologia; Unidades de Terapia Intensiva Neonatal; Indicadores de Morbimortalidade; Near Miss.

\begin{abstract}
Objective: Verifying the incidence of neonatal near miss (NNM), an indicator of "near-death" due to pre or postnatal complications, in a medium-sized maternity hospital in the Northeast of Brazil. Study model: retrospective cohort, with an inductive approach and descriptive procedures. Method: Birth charts were analyzed during January 2016, and NNM cases were identified according to the following criteria: gestational age < 32 weeks, newborn (NB) requiring resuscitation or depressed maneuver, birth weight $<1500 \mathrm{~g}$, need for care in a neonatal intensive care unit (NICU), need for mechanical ventilation, Apgar score at 1 and 5 minutes $\leq 7$. To verify the association between maternal variables and NMN diagnosis the Student's t-test was used. Results: 120 NB were evaluated. Among them, 26 born of normal birth and 94 of cesarean delivery. The mean maternal age was 26.61 ( \pm 7.9 ). The majority of the mothers had cardiovascular diseases (71.7\%) and $70.8 \%$ had a prenatal visit with several consultations below seven. An incidence of $30 \%$ of NNM was observed, most of them male (55\%). The hospitalization rate in the NICU was $17.5 \%$. The lower number of live children and higher mean cesarean births had a significant association $(p<0.05)$ with the NNM. Conclusion: The NNM indicator presented a high incidence, even in a reference hospital for a high-risk pregnancy, being a highly relevant method for the evaluation and prevention of severe morbidity in the NB.
\end{abstract}

Keywords: Neonatology; Intensive Care Units, Neonatal; Indicators of Morbidity and Mortality; Near Miss, Healthcare.

1. Docente do curso de Enfermagem da Universidade Federal de Campina Grande (UFCG), Campina Grande (PB), Brasil.

2. Graduada em Enfermagem pela Faculdade de Ciências Médicas de Campina Grande, Campina Grande (PB), Brasil.

3. Médico residente de Clínica Médica do Hospital Universitário Alcides Carneiro (HUAC) da UFCG, Campina Grande (PB), Brasil.

Lucian Batista de Oliveira. Universidade Federal de Campina Grande. Rua Carlos Chagas, s/n - São José. CEP: 58400-398. Campina Grande (PB), Brasil. lucianbaptist@gmail.com | Recebido em: 25/09/2018 | Aprovado em: 12/02/2020 


\section{INTRODUÇÃO}

$\mathrm{O}$ Brasil, seguindo a tendência mundial, apresentou redução na mortalidade infantil, entretanto, uma força de declínio semelhante não foi constatada na mortalidade neonatal, sugerindo assim, falhas na qualidade da assistência obstétrica e ao neonato ${ }^{1,2}$. O componente neonatal da mortalidade infantil reflete a qualidade dos serviços ofertados às mães e aos recém-nascidos (RN) durante os períodos antenatal, de parto e neonatal. As adequadas assistências pré-gravídica, gestacional, intraparto e aos RN são consideradas intervenções insubstituíveis à promoção da saúde neonatal, contudo, as profundas desigualdades nacionais e regionais, o acesso limitado aos cuidados necessários, a iniquidade na distribuição de leitos intensivos neonatais e a fragilidade dos serviços prestados, contribuem para o fortalecimento dos fatores de risco de morte dos neonatos ${ }^{1-3}$.

O número insuficiente de consultas de pré-natal está diretamente ligado ao baixo peso ao nascer, à prematuridade e ao óbito precoce ${ }^{4,5}$. A morbimortalidade por causas evitáveis, que são reduzíveis por adequado atendimento à gestante, é o principal componente da mortalidade neonatal evitável, seguido pela adequada atenção ao RN e pelo atendimento no parto ${ }^{5,6}$. Assim, o pré-natal e a garantia do acesso e da qualidade do atendimento, além da quantidade de consultas, são pontos-chave na meIhoria da atenção em prol da gestante e do $\mathrm{RN}^{6,7}$.

Desde a década de 1970, o conceito de near miss vem sendo cada vez mais usado na saúde como ferramenta para avaliar e melhorar a qualidade dos atendimentos perinatais e estimular a busca por mais indicadores que vão além da mortalidade neonatal, como no contexto de condições críticas, exemplificadas por casos graves de icterícia, encefalopatia, ou "síndrome de quase morte súbita", colaborando de forma relevante para um atendimento integral e livre de danos ${ }^{8-10}$.

Da mesma forma, o conceito de near miss neonatal (NMN) se refere a um RN que apresentou uma complicação grave, classificada dentro de critérios de avaliação de morbidade severa, até sete dias após o parto, mas que sobreviveu durante tal período ${ }^{8,11}$.
Ainda não há, entretanto, consenso em relação aos critérios que caracterizam um caso de $\mathrm{NMN}^{1,8}$. Uma revisão sistemática, em 2015, apontou os seguintes fatores como classificadores de NMN: peso ao nascer $<1750 \mathrm{~g}$, escore de Apgar $<7 \mathrm{em}$ 5 minutos, idade gestacional $<33$ semanas completas, terapia antibiótica parenteral (até 7 dias e antes de 28 dias), uso de Continuous Positive Airway Pressure (CPAP) nasal, qualquer intubação até 7 dias e antes de 28 dias de vida, fototerapia dentro das primeiras $24 \mathrm{~h}$ de vida, ressuscitação cardiopulmonar, uso de drogas vasoativas, uso de anticonvulsivantes, utilização de surfactante, uso de hemoderivados, uso de esteroides para tratamento de hipoglicemia refratária e cirurgia ${ }^{8}$. 0 mesmo estudo ainda sugeriu o teste dos seguintes critérios de manejo em estudos futuros: uso de esteroide pré-natal, nutrição parenteral, identificação de malformação congênita e admissão em unidade de terapia intensiva neonatal (UTIN), além da análise da pontuação de Apgar quando considerado um caso de NMN por outro critério ${ }^{8}$. Modelos de regressão logística, baseados em estudo de base hospitalar brasileiro, testaram 19 variáveis como possíveis critérios de NMN, observando-se associação significativa de cinco delas com o alto risco de morte neonatal: peso ao nascer $<1.500 \mathrm{~g}$, escore de Apgar no $5^{\circ}$ minuto de vida $<7$, uso de ventilação mecânica, idade gestacional $<32$ semanas e relato de malformações congênitas ${ }^{1}$.

O presente estudo teve como objetivo calcular a incidência de NMN em uma maternidade de médio porte no Nordeste brasileiro, caracterizando os casos quanto às características maternas e do pré-natal e as variáveis biológicas e de nascimento do RN, através da mensuração de critérios diagnósticos de NMN.

\section{MÉTODOS}

Trata-se de um estudo de coorte retrospectivo, com abordagem indutiva e procedimentos descritivos, realizado no Instituto de Saúde Elpídio de Almeida (ISEA), referência no atendimento materno-infantil de Campina Grande, município onde está localizado, e de grande parte do estado da Paraíba. 
O estudo foi desempenhado através da observação indireta, utilizando fonte de dados secundária.

A população alvo do estudo foi constituída por todos os nascidos após gestação de alto risco na referida maternidade com período de seguimento até a alta hospitalar, no mês de janeiro de 2016. Foi considerado como critério de inclusão todos aqueles neonatos com registros completos de indicadores neonatais, sendo utilizados como critérios de exclusão a ilegibilidade dos prontuários. O prontuário foi considerado ilegível quando dois diferentes pesquisadores assim o julgaram.

$O$ instrumento de coleta de dados consistiu em um formulário específico desenvolvido pelos pesquisadores, com as seguintes variáveis: sexo do RN (masculino ou feminino), idade gestacional (pré-termo, a termo e pós-termo), tipo de parto (cesáreo ou normal), número de consultas pré-natal ( $\leq 7$ ou $>7$ ), número de gestações prévias ( $\leq 2$ ou $>2$ ), desfecho neonatal (alta ou interno na UTIN), características da alta (alta médica, transferência, evasão ou óbito) e NMN (presente ou ausente), baseado nas informações contidas no prontuário durante período de internação.

Os critérios definidores de NMN utilizados foram baseados em estudo de coorte nacional brasileiro de base hospitalar ${ }^{1}$ e em revisão sistemática de literatura, que procurou estabelecer critérios empregados em estudos anteriores ou sugeridos para estudos futuros ${ }^{8}$, visto que apenas os critérios pragmáticos (peso ao nascer, idade gestacional e escore de Apgar no quinto minuto), embora utilizados em várias definições validadas, parecem não apontar claramente os marcadores de manejo do RN. Assim, foram selecionados os critérios: idade gestacional < 32 semanas; RN deprimido e/ou que necessitou de manobra de reanimação; peso ao nascimento < $1500 \mathrm{~g}$; necessidade de cuidados em terapia intensiva; necessidade de ventilação mecânica; escore de Apgar no $1^{\circ}$ e $5^{\circ}$ minutos $<7$.

Os resultados obtidos foram registrados em banco de dados eletrônico da versão 17.0 do software SPSS $^{\circledR}$, por meio de digitação dupla e posterior análise de consistência dos dados. As variáveis foram descritas através de frequências relativa e absoluta. Para verificar a associação entre as variáveis maternas e NMN foi utilizado o teste t de Student (nível de significância de 5\%). Foram calculadas proporções para as variáveis categóricas, média e desvio-padrão (DP) para as variáveis contínuas e a taxa de NMN por mil nascidos vivos (quociente entre o número de casos de near miss e o total de nascidos vivos).

Os dados foram apresentados através de proporções, médias e desvios-padrão (DP), bem como através de tabelas de contingência.

O presente estudo foi aprovado pelo Comitê de Ética em Pesquisa da Faculdade de Ciências Médicas de Campina Grande, conforme número de protocolo 72236917.5.0000.5175, encontrando-se de acordo com as normas que norteiam as pesquisas com seres humanos, sendo garantido o sigilo de informações através do anonimato e da confidencialidade dos participantes.

\section{RESULTADOS}

No período do estudo, foram admitidas 277 gestantes de alto risco, as quais tiveram os prontuários avaliados, sendo incluídas no estudo apenas 118 , que apresentavam preenchimento completo e legível. Duas das gestações eram gemelares, perfazendo uma amostra total de 120 RN. Vinte e seis $(21,7 \%)$ nasceram de parto vaginal e $94(78,3 \%)$ evoluíram para parto cesáreo.

A média de idade das genitoras foi de 26,61 anos $( \pm 7,29)$. Dentre as morbidades apresentadas que as caracterizaram como gestação de alto risco, as mais comuns foram doenças cardiovasculares presentes em $71,7 \%$ das mães, seguido de transtornos endócrinos, como o diabetes mellitus gestacional, encontrado em 13,3\%. Quando se avaliou o pré-natal, verificou-se que $29,9 \%$ apresentaram número de consultas superior a sete. Havia registro de abortamento prévio no prontuário de dez mães.

A maioria dos RN avaliados era do sexo masculino ( $55 \%$ do total). Um total de $17,5 \%$ foram admitidos em UTIN. Ao se analisar os indicadores neonatais selecionados, observou-se uma incidência de 300 casos de NMN/1000 nascidos vivos (Tabela 1 ). 


\section{Tabela 1}

Caracterização materna e neonatal da amostra durante o período de janeiro de 2017 no Instituto de Saúde Elpídio de Almeida, Campina Grande - Paraíba.

\begin{tabular}{|c|c|c|}
\hline Variáveis & $\mathrm{N}$ & $\%$ \\
\hline \multicolumn{3}{|l|}{ Sexo do RN } \\
\hline Masculino & 66 & 55,0 \\
\hline Feminino & 54 & 45,0 \\
\hline \multicolumn{3}{|l|}{ Idade gestacional } \\
\hline Pré-termo & 59 & 49,2 \\
\hline A termo & 60 & 50,0 \\
\hline Pós-termo & 1 & 8 \\
\hline \multicolumn{3}{|l|}{ Tipo de parto } \\
\hline Cesáreo & 94 & 78,3 \\
\hline Normal & 26 & 21,7 \\
\hline \multicolumn{3}{|c|}{ Número de consultas pré-natal } \\
\hline$\leq 7$ & 85 & 70,8 \\
\hline$>7$ & 35 & 29,2 \\
\hline \multicolumn{3}{|l|}{ Gestações prévias } \\
\hline$\leq 2$ & 69 & 57,5 \\
\hline$>2$ & 51 & 42,5 \\
\hline \multicolumn{3}{|l|}{ Desfecho neonatal } \\
\hline Alta & 99 & 82,5 \\
\hline Interno na UTIN & 21 & 17,5 \\
\hline \multicolumn{3}{|c|}{ Característica da alta } \\
\hline Alta médica & 113 & 94,2 \\
\hline Transferências & 5 & 4,2 \\
\hline Evasão & 1 & 0,8 \\
\hline Óbito & 1 & 0,8 \\
\hline \multicolumn{3}{|l|}{ Near miss neonatal } \\
\hline $\operatorname{Sim}$ & 36 & 30,0 \\
\hline Não & 84 & 70,0 \\
\hline
\end{tabular}

Em relação à frequência dos critérios de NMN utilizados, constatou-se que a necessidade de ventilação mecânica e de manobras de reanimação neonatal foram os mais presentes, sendo ambos observados em $22,5 \%$ dos prontuários avaliados. O escore de Apgar $<7$ no primeiro minuto esteve presente em $20,8 \%$ da amostra, porém, apenas $8,3 \%$ apresentaram o critério re- ferente a tal escore positivo, por se manterem abaixo de 7 no $5^{\circ}$ minuto (Figura 1 ).

Ao se avaliar as características maternas, comparando-se os grupos com e sem NMN, observou-se associação estatisticamente significativa $(p<0,05)$ entre maior número de filhos vivos e maior média de partos cesáreos com o grupo que apresentou NMN (Tabela 2). 


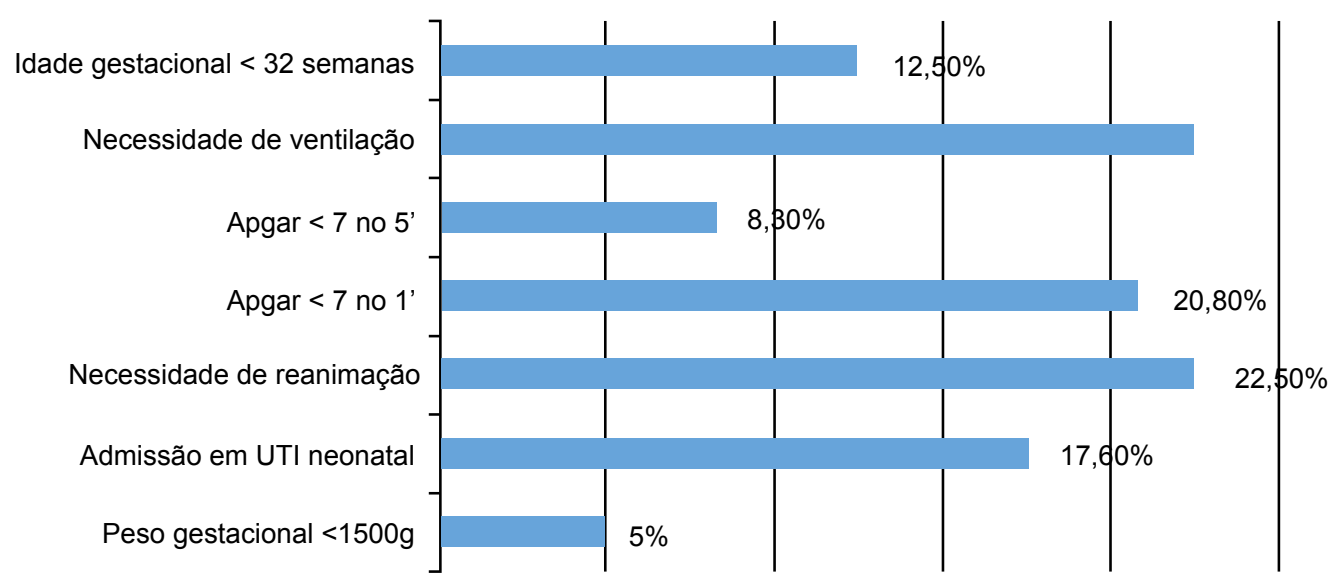

Figura 1: Distribuição dos critérios para near miss neonatal. Instituto de Saúde Elpídio de Almeida, Campina Grande Paraíba. 2017.

\section{Tabela 2}

Caracterização materna de acordo com o near miss neonatal durante o período de avaliação. Instituto de Saúde Elpídio de Almeida, Campina Grande - Paraíba. 2017.

\begin{tabular}{lccc}
\hline \multirow{2}{*}{ Variáveis } & \multicolumn{2}{c}{ Near miss } & p-valor* \\
\cline { 2 - 4 } & $\begin{array}{c}\text { Presente } \\
\text { Média }( \pm \mathrm{DP})\end{array}$ & $\begin{array}{c}\text { Ausente } \\
\text { Média ( } \pm \mathrm{DP})\end{array}$ \\
\hline Gestações Prévias & $2,19( \pm 1,50)$ & $2,46( \pm 1,36)$ & 0,360 \\
Filhos Vivos & $0,89( \pm 0,88)$ & $1,36( \pm 1,13)$ & $\mathbf{0 , 0 1 7}$ \\
Número de Consultas & $6,31( \pm 3,29)$ & $5,62( \pm 3,37)$ & 0,303 \\
Número de Partos anteriores & $1,06( \pm 1,09)$ & $1,32( \pm 1,23)$ & 0,244 \\
Número de Partos Vaginais & $0,86( \pm 1,04)$ & $0,87( \pm 1,19)$ & 0,971 \\
Número de Partos Cesáreas & $0,42( \pm 0,62)$ & $0,22( \pm 0,42)$ & $\mathbf{0 , 0 5 0}$ \\
Tempo de internação & $5,22( \pm 5,03)$ & $5,45( \pm 3,60)$ & 0,805 \\
\hline
\end{tabular}

*Teste T de Student

\section{DISCUSSÕES}

A proposta de inserção de um indicador de qualidade dos cuidados de saúde, como o near miss, auxilia no atendimento de alta qualidade no período neonatal, reduzindo a morbidade e podendo ser utilizado para monitorizar os neonatos que sofreram "quase morte".

Em um estudo nacional de base hospitalar, peso ao nascer, nascimento pré-termo, Apgar no $5^{\circ}$ minuto de vida, ventilação mecânica e presença de malformação congênita, fortes preditores de óbito neonatal, foram considerados critérios válidos para definição de NMN, sendo ventilação mecânica o critério mais frequente ${ }^{1}$. No presente estudo, $30 \%$ dos RN possuíam pelo menos um dos critérios para clas- sificação de caso de NMN, sendo os mais frequentes as necessidades de ventilação e reanimação.

O nascimento precoce é responsável por comprometimentos biológicos potencialmente fatais no período neonatal. No estudo nacional em que se analisou 24.197 puérperas e seus RN, constatou-se que o risco de morte foi maior para aqueles com peso ao nascer menor que $1.500 \mathrm{~g}$ e a idade gestacional menor que 32 semanas $^{1}$. Em consonância, em outro estudo brasileiro e multicêntrico, que avaliou a mortalidade neonatal até 27 dias de vida - em unidades de alto risco, o baixo peso ao nascer e a prematuridade também foram marcadores de mortalidade ${ }^{9}$. Percentual relevante da amostra do presente estudo apresentavam esses dois marcadores. 
Um estudo nacional de coorte prospectiva de base hospitalar, diante dos fatores associados ao óbito neonatal nas primeiras 24 horas de vida, analisou três variáveis prevalentes: as ligadas ao atendimento perinatal, as variáveis biológicas (peso ao nascer e sexo masculino) e as que refletem no atendimento ao parto (Apgar no quinto minuto). Observou-se que quanto menor a idade gestacional, maior o risco de NMN, destacando-se a presença de complicações orgânicas associadas à prematuridade e associação entre maior idade gestacional e maior o índice de sobrevida do neonato ${ }^{12}$.

O maior número de partos cesáreos esteve associado a maior risco de NMN neste estudo. Analogamente, em um estudo transversal realizado na região Sul do país, dentre as variáveis relativas à gestação, ao parto e à assistência recebida, o parto cesáreo e realizado em hospital público associou-se de forma significativa ao desfecho de $\mathrm{NMN}^{13}$. Deve ser considerada a possibilidade desta associação estar relacionada a gravidezes de mais alto risco, que culminavam com maior frequência de indicação de cesáreas e também com maior prevalência de complicações neonatais.

Quanto às limitações do estudo, podemos incluir o período de coleta, pois, não havendo no serviço prontuários informatizados, o tempo para coleta foi superior ao esperado, contribuindo também para isso o fato do hospital não dispor de profissionais suficientes para acompanhamento do pesquisador durante períodos ampliados de coleta de dados. Além disso, o número elevado de prontuários incompatíveis com as informações necessárias para a coleta representou uma das maiores dificuldades na identificação de casos de near miss, apontando uma necessidade premente de educação continuada nas instituições de atendimento ao RN no sentido de estimular o preenchimento adequado.

Ademais, embora a escolha dos critérios utilizados na definição de NMN no presente estudo tenha embasamento na literatura científica sobre o tema, a definição proposta ainda não foi validada. A população de estudo foi relativamente pequena e ocorreu apenas um óbito neonatal, considerado padrão-ouro nos demais estudos de validação. Nesse sentido a Organização Mundial da Saúde (OMS) propôs um protocolo com vários critérios como marcadores de $\mathrm{NMN}^{14}$, classifica- dos em categorias de indicadores de disfunção orgânica, clínicos ou laboratoriais, indicadores de manejo de gravidade e condições dos RN associadas à maior gravidade no período neonatal ${ }^{14,15}$.

A relevância deste estudo parte do pressuposto de que, ao ser verificada a incidência de NMN, se desperte a atenção de gestores e profissionais de saúde para uma assistência neonatal mais eficaz, reduzindo a morbidade e consequentemente sequelas físicas, cognitivas e emocionais para o binômio mãe-filho, implementando o indicador NMN na prática clínica como um instrumento de avaliação de risco prático, pouco dispendioso e efetivo, capaz de contribuir na redução da mortalidade infantil, bem como na identificação precoce de riscos para o óbito neonatal.

\section{CONCLUSÕES}

Os resultados apontam que o indicador de NMN apresentou uma elevada incidência, mesmo se tratando de um hospital de referência para atendimento à gestação de alto risco. Além disso, observa-se uma baixa adesão ao pré-natal e número de partos cesáreos bem superiores ao vaginal. Dentre os critérios diagnósticos de near miss, as necessidades de reanimação e de ventilação mecânica figuram como os sinais prevalentes na população estudada.

Pode-se ainda destacar a relevância do indicador como um método capaz de prevenir a "quase morte" do RN, auxiliando no manejo adequado e atendimento rápido e preciso, fatores imprescindíveis para obtenção de resultados positivos no desfecho dos casos de NMN, evitando assim, a morte neonatal.

\section{REFERÊNCIAS}

1. Silva AAM, Leite ÁJM, Lamy ZC, Moreira MEL, Gurgel RQ, Cunha AJLA et al. Morbidade neonatal near miss na pesquisa Nascer no Brasil. Cad. Saúde Pública [Internet]. 2014; 30(Suppl 1): S182-S191.

2. Tomasi E, Fernandes PAA, Fischer T, Siqueira FCV, Silveira DS, Thumé E et al. Qualidade da atenção pré-natal na rede básica de saúde do Brasil: indicadores e desigualdades sociais. Cad. Saúde Publica [online]. 2017; 33(3): e00195815. 
3. McKinnon B, Harper S, Kaufman JS, Bergevin Y. Socioeconomic inequality in neonatal mortality in countries of low and middle income: a multicountry analysis. Lancet Glob Health. 2014; 2(3): e165-e173.

4. United Nations Children's Fund (UNICEF). Committing to Child Survival: A Promise Renewed Progress Report 2013. New York: UNICEF; 2014. Disponível em: http://www.unicef.org/publications/files/APR_Progress_Report_2013_9_ Sept_2013.pdf.

5. Basso CG, Neves ET, Silveira A. Associação entre realização de pré-natal e morbidade neonatal. Texto contexto enferm. 2012; 21(2):269-76.

6. Nascimento RM, Leite AJM, Almeida NMGS, Almeida PC, Silva CF. Determinantes da mortalidade neonatal: estudo caso-controle em Fortaleza, Ceará, Brasil. Cad. Saúde Pública [online]. 2012; 28(3):559-72.

7. Fagundes $D Q$, Oliveira $A E$. Educação em saúde no pré-natal a partir do referencial teórico de Paulo Freire. Trab. educ. saúde [online]. 2017; 15(1):223-43.

8. Santos JP, Cecatti JG, Serruya SJ, Almeida PV, Duran P, Mucio $B$ et al. Neonatal Near Miss: the need for a standard definition and appropriate criteria and the rationale for a prospective surveillance system. Clinics [online]. 2015; 70(12):820-26.

9. Silva CF, Leite AJM, Almeida NMGS, Leon ACMP, Oloin I. Fatores associados ao óbito neonatal de recém-nascidos de Alto Risco: estudo multicêntrico em Unidades Neo- natais de Alto Risco no Nordeste brasileiro. Cad. Saúde Pública [online]. 2014; 30(2):355-68.

10. Brasil DRPA. Morbidade neonatal near miss em hospitais terciários da cidade do Recife-PE [Dissertação de mestrado] [Internet]. Recife: Universidade Federal de Pernambuco; 2015. Disponível em: https://repositorio.ufpe.br/bitstream/123456789/14041/1/DISSERTAÇÃO\%20DANYELLE\%20RODRIGUES.pdf

11. Pillegi CC, Camelo JS, Pardoná GC, Mussi-Pinhata MM, Cecatti JG, Mori R et al. Development of criteria for identifying neonatal near-miss cases: analysis of two WHO multicountry cross-sectional studies. BJOG. 2014; 12(Suppl 1): 110-18.

12. Castro ECM, Leite AJM, Guinsburg R. Mortality in the first $24 \mathrm{~h}$ of very low birth weight preterm infants in the Northeast of Brazil. Rev. paul. pediatr. 2016; 34(1):106-13.

13. Silva GA, Rosa KA, Saguier ESF, Henning E, Mucha F, Cristina FS. Estudo de base populacional sobre a prevalência de near miss neonatal em município do Sul do Brasil: prevalência e fatores associados. Ver. Bras. Saude Mater. Infant. 2017; 17(1):159-67.

14. Souza JP, Gülmezoglu AM, Carroli G, Lumbiganon P, Qureshi Z; WHOMCS Research Group. The World Health Organization multicountry survey on maternal and newborn health: study protocol. BMC Health Serv Res. 2011; 11:286.

15. Kale PL, Jorge MHPM, Laurenti R, Fonseca SC, Silva KS. Critérios Pragmáticos da definição do Near Miss Neonatal: um estudo comparativo. Rev. Saúde Pública. 2017; 51:111. 